\title{
Activation of Carbon Dioxide by Bicyclic
}

\section{Amidines}

Eduardo R. Pérez, * Regina H. A. Santos, Maria T. P. Gambardella, Luiz G. Macedo, Ubirajara P. Rodrigues-Filho, Jean-Claude Launay ${ }^{a}$ and Douglas W. Franco*

Instituto de Química de São Carlos, Universidade de São Paulo, C. P. 780, CEP 13560-970, São Carlos-SP, Brasil.

a Institut de Chimie de la Matière Condensée de Bordeaux, CNRS UPR 9048, F-33608 Pessac,

France

\section{Content}

1. Crystal data and details of the structure determination (Table 1S)

2. X-ray bond distances and angles for amidinium bicarbonates (Table 2S)

3. Geometrical parameters of $\mathrm{DBU}$ and $\mathrm{DBU}-\mathrm{CO}_{2}($ Table $3 \mathrm{~S})$

4. Geometrical parameters of PMDBD and PMDBD-CO $($ Table 4S)

5. Natural charges on atoms of DBU, PMDBD and their complexes with $\mathrm{CO}_{2}$ (Table 5S)

6. Cartesian coordinates and total energies for the calculated molecules

7. General analytical details and compounds characterization

\footnotetext{
* Corresponding authors: Tel./Fax: + 55163373 9976. E-mail: (D.W. Franco) douglas@iqsc.sc.usp.br; (E.R. Pérez) ed5bras@mailcity.com
} 
TABLE 1S. Crystal Data and Details of the Structure Determination

\begin{tabular}{|c|c|c|}
\hline & Bis-[DBUH] $]^{+} \mathrm{HCO}_{3}^{-}$ & Bis-[PMDBDH $]^{+} \mathrm{HCO}_{3}^{-}$ \\
\hline Formula & $\mathrm{C}_{9} \mathrm{H}_{17} \mathrm{~N}_{2} . \mathrm{HCO}_{3}$ & $\mathrm{C}_{13} \mathrm{H}_{25} \mathrm{~N}_{2} \cdot \mathrm{HCO}_{3} \cdot \mathrm{CHCl}_{3}$ \\
\hline Formula Weight & 214.26 & 380.87 \\
\hline Crystal System & Monoclinic & Monoclinic \\
\hline Space group & $\mathrm{P} 2{ }_{1} / \mathrm{n}$ & $\mathrm{C} 2 / \mathrm{c}$ \\
\hline $\mathrm{a}[\AA]$ & $7.229(1)$ & $24.276(1)$ \\
\hline $\mathrm{b}[\AA]$ & $18.385(2)$ & $9.1800(6)$ \\
\hline$c[\AA]$ & $8.1985(5)$ & $18.139(1)$ \\
\hline$\beta\left[^{\circ}\right]$ & $91.401(9)$ & $97.552(3)$ \\
\hline $\mathrm{V}\left[\AA^{3}\right]$ & $1089.3(2)$ & $4007.3(4)$ \\
\hline $\mathrm{Z}$ & 4 & 8 \\
\hline $\mathrm{D}($ calc $)\left[\mathrm{Mg} / \mathrm{m}^{3}\right]$ & 1.306 & 1.263 \\
\hline$\mu(\operatorname{MoK} \alpha)[/ \mathrm{mm}]$ & 0.097 & 0.437 \\
\hline $\mathrm{F}(000)$ & 464 & 1614 \\
\hline Crystal Size $[\mathrm{mm}]$ & $0.15 \times 0.20 \times 0.25$ & $0.14 \times 0.18 \times 0.22$ \\
\hline Temperature $(\mathrm{K})$ & 273 & 200 \\
\hline Radiation MoK $\alpha[\AA]$ & 0.71073 & 0.71073 \\
\hline$\theta$ min: $\max \left[^{\circ}\right]$ & $2.7: 30.0$ & $2.6,25.3$ \\
\hline Dataset hkl & $\overline{10}: 0 ; 0: 25 ; \overline{11}: 11$ & $\overline{26}: 29 ; \overline{8}: 11 ; \overline{21}: 21$ \\
\hline Tot., Uniq. Data, R(int) & $3387,3166,0.034$ & $11136,3608,0.043$ \\
\hline Obs. data $[\mathrm{I}>2.0 \sigma(\mathrm{I})]$ & 2311 & 2628 \\
\hline Nref, Npar & 3166,136 & 3608,289 \\
\hline $\mathrm{R}, \mathrm{wR} 2, \mathrm{~S}$ & $0.0558,0.1734,1.04$ & $0.0666,0.2245,1.03$ \\
\hline Min. and Max. Resd. Dens. [e/ $\left./ \AA^{3}\right]$ & $0.32,0.30$ & $0.26,0.53$ \\
\hline
\end{tabular}


TABLE 2S. Bond Distances $(\AA)$ and Angles $\left(^{\circ}\right)$ for $b i s-[\mathrm{DBUH}]^{+} \mathrm{HCO}_{3}{ }^{-}$and $b i s-[\mathrm{PMDBDH}]^{+} \mathrm{HCO}_{3}{ }^{-}$

\begin{tabular}{|c|c|c|c|}
\hline \multicolumn{2}{|c|}{$[\mathrm{DBUH}]^{+} \mathrm{HCO}_{3}^{-}$} & \multicolumn{2}{|c|}{$[\mathrm{PMDBDH}]^{+} \mathrm{HCO}_{3}^{-}$} \\
\hline $\mathrm{O} 1-\mathrm{C}$ & $1.287(2)$ & O1-C14 & $1.256(3)$ \\
\hline $\mathrm{O} 2-\mathrm{C}$ & $1.252(2)$ & $\mathrm{O} 2-\mathrm{C} 14$ & $1.340(3)$ \\
\hline O3-C & $1.303(2)$ & O3-C14 & $1.245(3)$ \\
\hline $\mathrm{N} 1-\mathrm{C} 1$ & $1.461(3)$ & N1-C8 & $1.309(3)$ \\
\hline N1-C9 & $1.311(2)$ & N1-C7 & $1.485(3)$ \\
\hline $\mathrm{N} 2-\mathrm{C} 3$ & $1.473(2)$ & N2-C8 & $1.318(3)$ \\
\hline N2-C9 & $1.326(2)$ & $\mathrm{N} 2-\mathrm{C} 1$ & $1.478(3)$ \\
\hline $\mathrm{N} 2-\mathrm{C} 4$ & $1.475(2)$ & $\mathrm{C} 1-\mathrm{C} 2$ & $1.537(4)$ \\
\hline $\mathrm{C} 1-\mathrm{C} 2$ & $1.485(3)$ & C1-C9 & $1.511(4)$ \\
\hline $\mathrm{C} 2-\mathrm{C} 3$ & $1.505(3)$ & C1-C10 & $1.511(4)$ \\
\hline C4-C5 & $1.517(2)$ & C2-C3 & $1.530(5)$ \\
\hline C5-C6 & $1.529(3)$ & C3-C4 & $1.534(4)$ \\
\hline C6-C7 & $1.519(2)$ & C4-C13 & $1.536(5)$ \\
\hline $\mathrm{C} 7-\mathrm{C} 8$ & $1.532(2)$ & $\mathrm{C} 4-\mathrm{C} 5$ & $1.533(5)$ \\
\hline C8-C9 & $1.499(2)$ & $\mathrm{C} 4-\mathrm{C} 8$ & $1.509(4)$ \\
\hline C1-N1-C9 & $123.3(1)$ & N1-C7-C11 & $107.1(2)$ \\
\hline C3-N2-C9 & $121.4(1)$ & N1-C8-N2 & $119.3(2)$ \\
\hline C4-N2-C9 & 121.7(1) & N1-C8-C4 & $120.1(2)$ \\
\hline C3-N2-C4 & 116.9(1) & N2-C1-C10 & $107.8(2)$ \\
\hline $\mathrm{N} 1-\mathrm{C} 1-\mathrm{C} 2$ & $110.1(2)$ & N2-C1-C9 & $107.2(2)$ \\
\hline $\mathrm{N} 1-\mathrm{C} 9-\mathrm{N} 2$ & $121.6(1)$ & N2-C1-C2 & $110.1(2)$ \\
\hline N1-C9-C8 & $117.5(1)$ & N2-C8-C4 & $120.5(2)$ \\
\hline N2-C3-C2 & 111.7(1) & C7-N1-C8 & $127.5(2)$ \\
\hline N2-C4-C5 & $113.5(1)$ & C1-N2-C8 & $129.0(2)$ \\
\hline N2-C9-C8 & $120.9(1)$ & C1-C2-C9 & $111.0(2)$ \\
\hline $\mathrm{C} 1-\mathrm{C} 2-\mathrm{C} 3$ & 111.1(2) & C9-C1-C10 & $109.2(3)$ \\
\hline C4-C5-C6 & $113.9(1)$ & $\mathrm{C} 2-\mathrm{C} 1-\mathrm{C} 10$ & $111.4(2)$ \\
\hline C5-C6-C7 & $114.1(1)$ & $\mathrm{C} 1-\mathrm{C} 2-\mathrm{C} 3$ & $111.8(2)$ \\
\hline C6-C7-C8 & 114.3(1) & C2-C3-C4 & 111.1(3) \\
\hline C7-C8-C9 & $115.4(1)$ & $\mathrm{C} 3-\mathrm{C} 4-\mathrm{C} 8$ & $108.5(2)$ \\
\hline $\mathrm{O} 2-\mathrm{C}-\mathrm{O} 3$ & 119.7(1) & $\mathrm{C} 8-\mathrm{C} 4-\mathrm{C} 13$ & $108.5(2)$ \\
\hline $\mathrm{O} 1-\mathrm{C}-\mathrm{O} 2$ & $121.4(1)$ & C5-C4-C13 & $110.2(3)$ \\
\hline $\mathrm{O} 1-\mathrm{C}-\mathrm{O} 3$ & $118.9(1)$ & C3-C4-C13 & $111.1(3)$ \\
\hline & & $\mathrm{C} 5-\mathrm{C} 4-\mathrm{C} 8$ & $107.6(2)$ \\
\hline & & C3-C4-C5 & $110.9(3)$ \\
\hline & & C4-C5-C6 & $111.3(3)$ \\
\hline & & C5-C6-C7 & $112.6(3)$ \\
\hline & & N1-C7-C6 & $110.7(2)$ \\
\hline & & $\mathrm{C} 11-\mathrm{C} 7-\mathrm{C} 12$ & $109.5(3)$ \\
\hline & & N1-C7-C12 & $106.0(2)$ \\
\hline & & C6-C7-C11 & $111.4(3)$ \\
\hline & & $\mathrm{C} 6-\mathrm{C} 7-\mathrm{C} 12$ & $111.9(3)$ \\
\hline & & O1-C14-O3 & $125.8(3)$ \\
\hline & & O2-C14-O3 & $116.2(2)$ \\
\hline & & O1-C14-O2 & $118.0(3)$ \\
\hline
\end{tabular}


TABLE 3S. Geometrical parameters of DBU and DBU- $\mathrm{CO}_{2}$ molecules (DBU values are in parenthesis)

\begin{tabular}{|c|c|c|c|}
\hline \multicolumn{2}{|l|}{ Bond Lengths (in $\AA$ ) } & \multicolumn{2}{|c|}{ All other C-H distances are $1.090 \AA}$. \\
\hline $\mathrm{O}(30)-\mathrm{C}(28)$ & 1.237 & $\mathrm{~N}(6)-\mathrm{C}(5)$ & $1.340(1.380)$ \\
\hline $\mathrm{O}(29)-\mathrm{C}(28)$ & 1.237 & $\mathrm{~N}(6)-\mathrm{C}(7)$ & $1.490(1.470)$ \\
\hline $\mathrm{N}(4)-\mathrm{C}(28)$ & 1.530 & $\mathrm{C}(7)-\mathrm{H}(18)$ & $1.070(1.100)$ \\
\hline $\mathrm{N}(4)-\mathrm{C}(5)$ & $1.330(1.290)$ & $\mathrm{C}(7)-\mathrm{C}(8)$ & $1.520(1.530)$ \\
\hline $\mathrm{N}(4)-\mathrm{C}(3)$ & $1.470(1.460)$ & $\mathrm{C}(8)-\mathrm{C}(9)$ & $1.530(1.530)$ \\
\hline $\mathrm{C}(3)-\mathrm{H}(16)$ & $1.080(1.090)$ & $\mathrm{C}(9)-\mathrm{C}(10)$ & $1.530(1.530)$ \\
\hline $\mathrm{C}(3)-\mathrm{C}(2)$ & $1.510(1.520)$ & $\mathrm{C}(10)-\mathrm{C}(11)$ & $1.530(1.540)$ \\
\hline$C(2)-C(1)$ & $1.510(1.520)$ & $\mathrm{C}(11)-\mathrm{H}(25)$ & $1.080(1.090)$ \\
\hline $\mathrm{C}(1)-\mathrm{H}(12)$ & $1.380(1.100)$ & $\mathrm{C}(11)-\mathrm{C}(5)$ & $1.520(1.520)$ \\
\hline $\mathrm{C}(1)-\mathrm{N}(6)$ & $1.480(1.470)$ & & \\
\hline \multicolumn{4}{|l|}{$\begin{array}{c}\text { Bond Angles } \\
\text { (in degrees) }\end{array}$} \\
\hline $\mathrm{O}(30)-\mathrm{C}(28)-\mathrm{N}(4)$ & 112.770 & $\mathrm{~N}(6)-\mathrm{C}(7)-\mathrm{H}(18)$ & $104.480(105.030)$ \\
\hline $\mathrm{O}(30)-\mathrm{C}(28)-\mathrm{O}(29)$ & 132.020 & $\mathrm{~N}(6)-\mathrm{C}(7)-\mathrm{H}(17)$ & $108.500(110.000)$ \\
\hline $\mathrm{C}(28)-\mathrm{N}(4)-\mathrm{C}(3)$ & 114.030 & $N(6)-C(7)-C(8)$ & $116.980(116.880)$ \\
\hline $\mathrm{C}(28)-\mathrm{N}(4)-\mathrm{C}(5)$ & 123.700 & $\mathrm{C}(7)-\mathrm{C}(8)-\mathrm{H}(19)$ & $107.390(107.620)$ \\
\hline $\mathrm{N}(4)-\mathrm{C}(3)-\mathrm{H}(16)$ & $107.760(108.010)$ & $\mathrm{C}(7)-\mathrm{C}(8)-\mathrm{H}(20)$ & $108.990(108.670)$ \\
\hline $\mathrm{N}(4)-\mathrm{C}(3)-\mathrm{H}(27)$ & 109.080(109.020) & $\mathrm{C}(7)-\mathrm{C}(8)-\mathrm{C}(9)$ & $113.400(114.430)$ \\
\hline $\mathrm{N}(4)-\mathrm{C}(3)-\mathrm{C}(2)$ & 109.360(112.930) & $\mathrm{C}(8)-\mathrm{C}(9)-\mathrm{H}(21)$ & 109.910(109.710) \\
\hline$C(3)-C(2)-C(1)$ & $109.130(108.580)$ & $\mathrm{C}(8)-\mathrm{C}(9)-\mathrm{H}(22)$ & $109.430(108.760)$ \\
\hline $\mathrm{C}(3)-\mathrm{C}(2)-\mathrm{H}(14)$ & $109.810(109.910)$ & $\mathrm{C}(8)-\mathrm{C}(9)-\mathrm{C}(10)$ & $111.470(112.880)$ \\
\hline $\mathrm{C}(3)-\mathrm{C}(2)-\mathrm{H}(15)$ & $110.510(111.368)$ & $\mathrm{C}(9)-\mathrm{C}(10)-\mathrm{H}(23)$ & $110.560(110.210)$ \\
\hline $\mathrm{C}(2)-\mathrm{C}(1)-\mathrm{N}(6)$ & $112.230(110.970)$ & $\mathrm{C}(9)-\mathrm{C}(10)-\mathrm{H}(24)$ & $109.650(109.370)$ \\
\hline $\mathrm{C}(2)-\mathrm{C}(1)-\mathrm{H}(13)$ & $110.550(110.690)$ & $\mathrm{C}(9)-\mathrm{C}(10)-\mathrm{C}(11)$ & $113.360(113.200)$ \\
\hline $\mathrm{C}(2)-\mathrm{C}(1)-\mathrm{H}(12)$ & $108.440(110.250)$ & $\mathrm{C}(10)-\mathrm{C}(11)-\mathrm{H}(25)$ & $110.590(109.850)$ \\
\hline $\mathrm{C}(1)-\mathrm{N}(6)-\mathrm{C}(5)$ & $121.550(118.240)$ & $\mathrm{C}(10)-\mathrm{C}(11)-\mathrm{H}(26)$ & $108.510(110.020)$ \\
\hline $\mathrm{N}(6)-\mathrm{C}(5)-\mathrm{N}(4)$ & $120.920(125.420)$ & $C(10)-C(11)-C(5)$ & $116.640(116.070)$ \\
\hline $\mathrm{C}(1)-\mathrm{N}(6)-\mathrm{C}(7)$ & $112.020(112.240)$ & & \\
\hline \multicolumn{4}{|l|}{$\begin{array}{l}\text { Dihedral Angles } \\
\text { (in degrees) }\end{array}$} \\
\hline $\mathrm{C}(5)-\mathrm{N}(4)-\mathrm{C}(28)-\mathrm{O}(30)$ & 130.400 & $\mathrm{~N}(6)-\mathrm{C}(5)-\mathrm{C}(11)-\mathrm{H}(26)$ & $-84.460(-70.410)$ \\
\hline $\mathrm{C}(5)-\mathrm{N}(4)-\mathrm{C}(28)-\mathrm{O}(29)$ & -52.170 & $\mathrm{~N}(6)-\mathrm{C}(5)-\mathrm{C}(11)-\mathrm{H}(25)$ & $162.590(176.070)$ \\
\hline $\mathrm{N}(6)-\mathrm{C}(5)-\mathrm{N}(4)-\mathrm{C}(28)$ & 171.700 & $\mathrm{~N}(6)-\mathrm{C}(7)-\mathrm{C}(8)-\mathrm{C}(9)$ & $-84.810(-84.450)$ \\
\hline $\mathrm{N}(6)-\mathrm{C}(5)-\mathrm{N}(4)-\mathrm{C}(3)$ & $-6.950(-2.220)$ & $\mathrm{N}(6)-\mathrm{C}(7)-\mathrm{C}(8)-\mathrm{H}(19)$ & $153.660(154.150)$ \\
\hline $\mathrm{C}(5)-\mathrm{N}(4)-\mathrm{C}(3)-\mathrm{C}(2)$ & $35.450(26.430)$ & $\mathrm{N}(6)-\mathrm{C}(7)-\mathrm{C}(8)-\mathrm{H}(20)$ & $39.530(40.040)$ \\
\hline C(5)-N(4)-C(3)-H(27) & $-86.560(-96.070)$ & $\mathrm{C}(7)-\mathrm{C}(8)-\mathrm{C}(9)-\mathrm{C}(10)$ & 40.590(42.090) \\
\hline $\mathrm{C}(5)-\mathrm{N}(4)-\mathrm{C}(3)-\mathrm{H}(16)$ & $156.990(149.220)$ & $\mathrm{C}(7)-\mathrm{C}(8)-\mathrm{C}(9)-\mathrm{H}(21)$ & $-80.960(-80.110)$ \\
\hline $\mathrm{N}(4)-\mathrm{C}(3)-\mathrm{C}(2)-\mathrm{H}(14)$ & $66.640(69.000)$ & $\mathrm{C}(7)-\mathrm{C}(8)-\mathrm{C}(9)-\mathrm{H}(22)$ & $162.380(164.130)$ \\
\hline N(4)-C(3)-C(2)-H(15) & $-174.770(-172.140)$ & $\mathrm{C}(8)-\mathrm{C}(9)-\mathrm{C}(10)-\mathrm{H}(23)$ & $-80.170(-83.640)$ \\
\hline $\mathrm{N}(4)-\mathrm{C}(3)-\mathrm{C}(2)-\mathrm{C}(1)$ & $-54.350(-50.920)$ & $\mathrm{C}(8)-\mathrm{C}(9)-\mathrm{C}(10)-\mathrm{H}(24)$ & $163.860(160.690)$ \\
\hline $\mathrm{C}(3)-\mathrm{C}(2)-\mathrm{C}(1)-\mathrm{H}(12)$ & $-68.200(-69.390)$ & $\mathrm{C}(8)-\mathrm{C}(9)-\mathrm{C}(10)-\mathrm{C}(11)$ & $43.560(39.000)$ \\
\hline $\mathrm{C}(3)-\mathrm{C}(2)-\mathrm{C}(1)-\mathrm{H}(13)$ & $168.210(172.470)$ & $C(9)-C(10)-C(11)-C(5)$ & $-86.840(-88.280)$ \\
\hline $\mathrm{C}(3)-\mathrm{C}(2)-\mathrm{C}(1)-\mathrm{N}(6)$ & $48.600(52.820)$ & $\mathrm{C}(9)-\mathrm{C}(10)-\mathrm{C}(11)-\mathrm{H}(26)$ & $37.420(36.250)$ \\
\hline $\mathrm{C}(2)-\mathrm{C}(1)-\mathrm{N}(6)-\mathrm{C}(5)$ & $-21.640(-31.540)$ & $\mathrm{C}(9)-\mathrm{C}(10)-\mathrm{C}(11)-\mathrm{H}(25)$ & $152.350(152.890)$ \\
\hline $\mathrm{C}(2)-\mathrm{C}(1)-\mathrm{N}(6)-\mathrm{C}(7)$ & $166.000(170.610)$ & & \\
\hline $\mathrm{C}(1)-\mathrm{N}(6)-\mathrm{C}(5)-\mathrm{N}(4)$ & $-0.630(5.100)$ & & \\
\hline $\mathrm{C}(1)-\mathrm{N}(6)-\mathrm{C}(5)-\mathrm{C}(11)$ & $-179.050(-178.260)$ & & \\
\hline $\mathrm{C}(1)-\mathrm{N}(6)-\mathrm{C}(7)-\mathrm{C}(8)$ & $-146.770(-154.910)$ & & \\
\hline $\mathrm{C}(1)-\mathrm{N}(6)-\mathrm{C}(7)-\mathrm{H}(18)$ & $-26.670(-35.130)$ & & \\
\hline $\mathrm{C}(1)-\mathrm{N}(6)-\mathrm{C}(7)-\mathrm{H}(17)$ & $86.550(78.520)$ & & \\
\hline $\mathrm{N}(6)-\mathrm{C}(5)-\mathrm{C}(11)-\mathrm{C}(10)$ & $40.970(54.440)$ & & \\
\hline
\end{tabular}


TABLE 4S. Geometrical parameters of PMDBD and PMDBD- $\mathrm{CO}_{2}$ molecules (PMDBD values are in parenthesis)

\begin{tabular}{|c|c|c|c|}
\hline \multirow{2}{*}{$\begin{array}{l}\text { Bond Lengths (in } \AA \text { ) } \\
\mathrm{O}(41)-\mathrm{C}(40)\end{array}$} & \multicolumn{3}{|c|}{ All other $\mathrm{C}-\mathrm{H}$ distances are $1.090 \AA}$. \\
\hline & 1.225 & $C(6)-C(5)$ & $1.530(1.530)$ \\
\hline $\mathrm{O}(42)-\mathrm{C}(40)$ & 1.240 & $\mathrm{C}(6)-\mathrm{C}(1)$ & $1.540(1.540)$ \\
\hline $\mathrm{N}(13)-\mathrm{C}(40)$ & 1.550 & $C(6)-C(9)$ & $1.550(1.540)$ \\
\hline $\mathrm{N}(13)-\mathrm{C}(12)$ & $1.527(1.475)$ & $\mathrm{C}(9)-\mathrm{H}(29)$ & $1.080(1.090)$ \\
\hline $\mathrm{N}(13)-\mathrm{C}(5)$ & $1.340(1.280)$ & $\mathrm{C}(1)-\mathrm{C}(2)$ & $1.520(1.520)$ \\
\hline $\mathrm{C}(12)-\mathrm{C}(15)$ & $1.540(1.540)$ & $C(2)-C(3)$ & $1.530(1.540)$ \\
\hline $\mathrm{C}(12)-\mathrm{C}(14)$ & $1.530(1.530)$ & $\mathrm{C}(3)-\mathrm{C}(8)$ & $1.530(1.530)$ \\
\hline $\mathrm{C}(12)-\mathrm{C}(11)$ & $1.530(1.540)$ & $\mathrm{C}(3)-\mathrm{C}(7)$ & $1.540(1.540)$ \\
\hline $\mathrm{C}(15)-\mathrm{H}(38)$ & $1.080(1.090)$ & $\mathrm{C}(7)-\mathrm{H}(22)$ & $1.080(1.090)$ \\
\hline $\mathrm{C}(14)-\mathrm{H}(35)$ & $1.080(1.090)$ & $\mathrm{N}(4)-\mathrm{C}(3)$ & $1.490(1.480)$ \\
\hline $\mathrm{C}(11)-\mathrm{C}(10)$ & $1.510(1.520)$ & $\mathrm{N}(4)-\mathrm{H}(20)$ & $1.020(1.020)$ \\
\hline \multirow[t]{2}{*}{$\mathrm{C}(10)-\mathrm{C}(6)$} & $1.530(1.540)$ & $\mathrm{N}(4)-\mathrm{C}(5)$ & $1.320(1.390)$ \\
\hline & & $\mathrm{H}(20)-\mathrm{O}(42)$ & 1.710 \\
\hline \multicolumn{4}{|l|}{$\begin{array}{l}\text { Bond Angles } \\
\text { (in degrees) }\end{array}$} \\
\hline $\mathrm{O}(41)-\mathrm{C}(40)-\mathrm{O}(42)$ & 130.010 & $\mathrm{C}(6)-\mathrm{C}(5)-\mathrm{N}(4)$ & $116.080(114.820)$ \\
\hline $\mathrm{O}(41)-\mathrm{C}(40)-\mathrm{N}(13)$ & 115.300 & $\mathrm{C}(6)-\mathrm{C}(1)-\mathrm{H}(16)$ & $108.180(108.840)$ \\
\hline $\mathrm{C}(40)-\mathrm{N}(13)-\mathrm{C}(5)$ & 120.340 & $\mathrm{C}(6)-\mathrm{C}(1)-\mathrm{H}(17)$ & $108.920(108.840)$ \\
\hline $\mathrm{C}(5)-\mathrm{N}(13)-\mathrm{C}(12)$ & $123.090(122.620)$ & $\mathrm{C}(6)-\mathrm{C}(9)-\mathrm{H}(27)$ & $112.160(111.770)$ \\
\hline $\mathrm{N}(13)-\mathrm{C}(12)-\mathrm{C}(11)$ & $110.680(113.790)$ & $\mathrm{C}(6)-\mathrm{C}(9)-\mathrm{H}(28)$ & $108.930(109.710)$ \\
\hline $\mathrm{N}(13)-\mathrm{C}(12)-\mathrm{C}(14)$ & $110.030(107.770)$ & $\mathrm{C}(6)-\mathrm{C}(9)-\mathrm{H}(29)$ & $112.370(111.970)$ \\
\hline $\mathrm{N}(13)-\mathrm{C}(12)-\mathrm{C}(15)$ & $108.970(106.850)$ & $C(6)-C(1)-C(2)$ & $112.680(112.900)$ \\
\hline $\mathrm{C}(12)-\mathrm{C}(15)-\mathrm{H}(37)$ & $109.330(110.850)$ & $\mathrm{C}(1)-\mathrm{C}(2)-\mathrm{C}(3)$ & $112.020(112.570)$ \\
\hline $\mathrm{C}(12)-\mathrm{C}(15)-\mathrm{H}(38)$ & $110.780(110.300)$ & $\mathrm{C}(1)-\mathrm{C}(2)-\mathrm{H}(18)$ & $110.330(110.090)$ \\
\hline $\mathrm{C}(12)-\mathrm{C}(15)-\mathrm{H}(39)$ & $111.410(111.450)$ & $\mathrm{C}(1)-\mathrm{C}(2)-\mathrm{H}(19)$ & $109.920(109.980)$ \\
\hline $\mathrm{C}(12)-\mathrm{C}(14)-\mathrm{H}(34)$ & $110.520(110.770)$ & $\mathrm{C}(2)-\mathrm{C}(3)-\mathrm{N}(4)$ & $110.940(108.710)$ \\
\hline $\mathrm{C}(12)-\mathrm{C}(14)-\mathrm{H}(35)$ & $112.280(110.730)$ & $\mathrm{C}(2)-\mathrm{C}(3)-\mathrm{C}(7)$ & $110.870(110.940)$ \\
\hline $\mathrm{C}(12)-\mathrm{C}(14)-\mathrm{H}(36)$ & $108.740(110.900)$ & $\mathrm{C}(2)-\mathrm{C}(3)-\mathrm{C}(8)$ & $110.890(110.130)$ \\
\hline $\mathrm{C}(12)-\mathrm{C}(11)-\mathrm{H}(32)$ & $109.670(109.330)$ & $\mathrm{C}(3)-\mathrm{C}(7)-\mathrm{H}(21)$ & $111.390(111.880)$ \\
\hline $\mathrm{C}(12)-\mathrm{C}(11)-\mathrm{H}(33)$ & $107.070(109.330)$ & $\mathrm{C}(3)-\mathrm{C}(7)-\mathrm{H}(22)$ & $110.890(110.470)$ \\
\hline $\mathrm{C}(12)-\mathrm{C}(11)-\mathrm{C}(10)$ & $113.180(112.000)$ & $\mathrm{C}(3)-\mathrm{C}(7)-\mathrm{H}(23)$ & $110.150(111.000)$ \\
\hline $\mathrm{C}(11)-\mathrm{C}(10)-\mathrm{C}(6)$ & $110.670(111.270)$ & $\mathrm{C}(3)-\mathrm{C}(8)-\mathrm{H}(24)$ & $109.950(110.690)$ \\
\hline $\mathrm{C}(11)-\mathrm{C}(10)-\mathrm{H}(30)$ & $110.620(111.810)$ & $\mathrm{C}(3)-\mathrm{C}(8)-\mathrm{H}(25)$ & $111.360(111.080)$ \\
\hline $\mathrm{C}(11)-\mathrm{C}(10)-\mathrm{H}(31)$ & $110.710(109.810)$ & $\mathrm{C}(3)-\mathrm{C}(8)-\mathrm{H}(26)$ & $111.050(110.780)$ \\
\hline$C(10)-C(6)-C(5)$ & $111.200(108.920)$ & $\mathrm{C}(3)-\mathrm{N}(4)-\mathrm{H}(20)$ & $116.090(112.060)$ \\
\hline $\mathrm{C}(10)-\mathrm{C}(6)-\mathrm{C}(1)$ & $110.040(110.660)$ & $\mathrm{C}(3)-\mathrm{N}(4)-\mathrm{C}(5)$ & $130.070(122.230)$ \\
\hline $\mathrm{C}(10)-\mathrm{C}(6)-\mathrm{C}(9)$ & $109.700(109.930)$ & $\mathrm{C}(6)-\mathrm{C}(5)-\mathrm{N}(13)$ & $122.700(126.460)$ \\
\hline
\end{tabular}




\begin{tabular}{|c|c|c|c|}
\hline $\begin{array}{l}\text { Dihedral Angles } \\
\text { (in degrees) }\end{array}$ & & & \\
\hline $\mathrm{O}(41)-\mathrm{C}(40)-\mathrm{N}(13)-\mathrm{C}(5)$ & 151.410 & $\mathrm{~N}(13)-\mathrm{C}(12)-\mathrm{C}(15)-\mathrm{H}(38)$ & $-58.640(-52.930)$ \\
\hline $\mathrm{O}(42)-\mathrm{C}(40)-\mathrm{N}(13)-\mathrm{C}(5)$ & -29.120 & $\mathrm{~N}(13)-\mathrm{C}(12)-\mathrm{C}(15)-\mathrm{H}(39)$ & $62.320(66.560)$ \\
\hline $\mathrm{C}(40)-\mathrm{N}(13)-\mathrm{C}(5)-\mathrm{C}(6)$ & 176.580 & $C(12)-C(11)-C(10)-C(6)$ & $60.380(57.300)$ \\
\hline $\mathrm{N}(13)-\mathrm{C}(5)-\mathrm{C}(6)-\mathrm{C}(10)$ & $25.160(19.140)$ & $\mathrm{C}(6)-\mathrm{C}(1)-\mathrm{C}(2)-\mathrm{C}(3)$ & $-55.810(-54.810)$ \\
\hline $\mathrm{N}(13)-\mathrm{C}(5)-\mathrm{C}(6)-\mathrm{C}(1)$ & $146.420(139.680)$ & $\mathrm{C}(6)-\mathrm{C}(1)-\mathrm{C}(2)-\mathrm{H}(18)$ & $-176.830(-176.500)$ \\
\hline $\mathrm{N}(13)-\mathrm{C}(5)-\mathrm{C}(6)-\mathrm{C}(9)$ & $-94.670(-100.520)$ & $\mathrm{C}(6)-\mathrm{C}(1)-\mathrm{C}(2)-\mathrm{H}(19)$ & $66.640(66.710)$ \\
\hline $\mathrm{C}(5)-\mathrm{C}(6)-\mathrm{C}(9)-\mathrm{H}(27)$ & $63.860(61.720)$ & $\mathrm{C}(1)-\mathrm{C}(2)-\mathrm{C}(3)-\mathrm{N}(4)$ & $33.790(47.820)$ \\
\hline $\mathrm{C}(5)-\mathrm{C}(6)-\mathrm{C}(9)-\mathrm{H}(28)$ & $-176.620(-178.630)$ & $\mathrm{C}(1)-\mathrm{C}(2)-\mathrm{C}(3)-\mathrm{C}(7)$ & $-84.630(-73.420)$ \\
\hline $\mathrm{C}(5)-\mathrm{C}(6)-\mathrm{C}(9)-\mathrm{H}(29)$ & $-56.810(-58.530)$ & $\mathrm{C}(1)-\mathrm{C}(2)-\mathrm{C}(3)-\mathrm{C}(8)$ & $153.190(165.550)$ \\
\hline $\mathrm{C}(5)-\mathrm{C}(6)-\mathrm{C}(1)-\mathrm{H}(16)$ & $177.620(173.430)$ & $\mathrm{C}(2)-\mathrm{C}(3)-\mathrm{C}(7)-\mathrm{H}(21)$ & $55.110(59.480)$ \\
\hline $\mathrm{C}(5)-\mathrm{C}(6)-\mathrm{C}(1)-\mathrm{H}(17)$ & $-67.150(-71.370)$ & $\mathrm{C}(2)-\mathrm{C}(3)-\mathrm{C}(7)-\mathrm{H}(22)$ & $175.390(179.190)$ \\
\hline $\mathrm{C}(5)-\mathrm{C}(6)-\mathrm{C}(10)-\mathrm{H}(30)$ & $-169.810(-168.580)$ & $\mathrm{C}(2)-\mathrm{C}(3)-\mathrm{C}(7)-\mathrm{H}(23)$ & $-65.070(-61.300)$ \\
\hline $\mathrm{C}(5)-\mathrm{C}(6)-\mathrm{C}(10)-\mathrm{H}(31)$ & $74.220(75.520)$ & $\mathrm{C}(2)-\mathrm{C}(3)-\mathrm{C}(8)-\mathrm{H}(24)$ & $58.800(61.250)$ \\
\hline$C(5)-C(6)-C(10)-C(11)$ & $-48.170(-45.820)$ & $\mathrm{C}(2)-\mathrm{C}(3)-\mathrm{C}(8)-\mathrm{H}(25)$ & $178.180(-179.200)$ \\
\hline$C(5)-C(6)-C(1)-C(2)$ & $55.900(50.980)$ & $\mathrm{C}(2)-\mathrm{C}(3)-\mathrm{C}(8)-\mathrm{H}(26)$ & $-61.000(-59.060)$ \\
\hline $\mathrm{C}(5)-\mathrm{N}(13)-\mathrm{C}(12)-\mathrm{C}(11)$ & $19.870(10.330)$ & $\mathrm{C}(2)-\mathrm{C}(3)-\mathrm{N}(4)-\mathrm{H}(20)$ & $171.100(-179.540)$ \\
\hline $\mathrm{C}(5)-\mathrm{N}(13)-\mathrm{C}(12)-\mathrm{C}(14)$ & $138.020(131.870)$ & $C(2)-C(3)-N(4)-C(5)$ & $-18.000(-45.370)$ \\
\hline $\mathrm{C}(5)-\mathrm{N}(13)-\mathrm{C}(12)-\mathrm{C}(15)$ & $-100.250(-111.550)$ & $C(3)-N(4)-C(5)-C(6)$ & $20.340(45.630)$ \\
\hline $\mathrm{N}(13)-\mathrm{C}(12)-\mathrm{C}(11)-\mathrm{H}(32)$ & $79.210(83.450)$ & $\mathrm{C}(3)-\mathrm{N}(4)-\mathrm{C}(5)-\mathrm{N}(13)$ & $-162.990(-138.440)$ \\
\hline $\mathrm{N}(13)-\mathrm{C}(12)-\mathrm{C}(11)-\mathrm{H}(33)$ & $-166.310(160.970)$ & $\mathrm{N}(4)-\mathrm{C}(5)-\mathrm{C}(6)-\mathrm{C}(1)$ & $-37.010(-44.850)$ \\
\hline $\mathrm{N}(13)-\mathrm{C}(12)-\mathrm{C}(14)-\mathrm{H}(34)$ & $-52.830(-61.360)$ & $\mathrm{N}(4)-\mathrm{C}(5)-\mathrm{N}(13)-\mathrm{C}(12)$ & $172.360(-176.400)$ \\
\hline $\mathrm{N}(13)-\mathrm{C}(12)-\mathrm{C}(14)-\mathrm{H}(35)$ & $68.640(58.420)$ & & \\
\hline $\mathrm{N}(13)-\mathrm{C}(12)-\mathrm{C}(14)-\mathrm{H}(36)$ & $-170.850(178.620)$ & & \\
\hline $\mathrm{N}(13)-\mathrm{C}(12)-\mathrm{C}(15)-\mathrm{H}(37)$ & $-178.600(-172.860)$ & & \\
\hline
\end{tabular}


TABLE 5S. Natural charges on atoms

\begin{tabular}{|c|c|c|c|c|c|c|c|}
\hline \multirow[t]{2}{*}{ Atoms } & \multirow[b]{2}{*}{ No. } & \multicolumn{2}{|l|}{ Molecules } & \multirow[t]{2}{*}{ Atoms } & \multirow[b]{2}{*}{ No. } & \multicolumn{2}{|l|}{ Molecules } \\
\hline & & DBU & DBU-CO ${ }_{2}$ & & & PMDBD & $\mathrm{PMDBD}-\mathrm{CO}_{2}$ \\
\hline $\mathrm{C}$ & 1 & -0.16201 & -0.16529 & $\mathrm{C}$ & 1 & -0.37133 & -0.37388 \\
\hline $\mathrm{C}$ & 2 & -0.40953 & -0.41120 & $\mathrm{C}$ & 2 & -0.38483 & -0.39149 \\
\hline $\mathrm{C}$ & 3 & -0.20042 & -0.17134 & $\mathrm{C}$ & 3 & 0.11965 & 0.13229 \\
\hline $\mathrm{N}$ & 4 & -0.62629 & -0.50408 & $\mathrm{~N}$ & 4 & -0.67439 & -0.58717 \\
\hline $\mathrm{C}$ & 5 & 0.48135 & 0.58997 & $\mathrm{C}$ & 5 & 0.49901 & 0.59998 \\
\hline $\mathrm{N}$ & 6 & -0.53488 & -0.44371 & $\mathrm{C}$ & 6 & -0.13496 & -0.11863 \\
\hline $\mathrm{C}$ & 7 & -0.16533 & -0.16861 & $\mathrm{C}$ & 7 & -0.57917 & -0.56965 \\
\hline $\mathrm{C}$ & 8 & -0.39942 & -0.40527 & $\mathrm{C}$ & 8 & -0.56311 & -0.56899 \\
\hline $\mathrm{C}$ & 9 & -0.38118 & -0.38337 & $\mathrm{C}$ & 9 & -0.55828 & -0.55908 \\
\hline $\mathrm{C}$ & 10 & -0.38757 & -0.38667 & $\mathrm{C}$ & 10 & -0.37332 & -0.37862 \\
\hline $\mathrm{C}$ & 11 & -0.42680 & -0.43819 & $\mathrm{C}$ & 11 & -0.39013 & -0.39176 \\
\hline $\mathrm{H}$ & 12 & 0.18347 & 0.20950 & $\mathrm{C}$ & 12 & 0.08860 & 0.14602 \\
\hline $\mathrm{H}$ & 13 & 0.20061 & 0.21843 & $\mathrm{~N}$ & 13 & -0.60067 & -0.53125 \\
\hline $\mathrm{H}$ & 14 & 0.19948 & 0.21504 & $\mathrm{C}$ & 14 & -0.55627 & -0.57787 \\
\hline $\mathrm{H}$ & 15 & 0.21391 & 0.23364 & $\mathrm{C}$ & 15 & -0.56130 & -0.58164 \\
\hline $\mathrm{H}$ & 16 & 0.19030 & 0.22502 & $\mathrm{H}$ & 16 & 0.20996 & 0.22328 \\
\hline $\mathrm{H}$ & 17 & 0.18454 & 0.20807 & $\mathrm{H}$ & 17 & 0.20129 & 0.21040 \\
\hline $\mathrm{H}$ & 18 & 0.18426 & 0.21669 & $\mathrm{H}$ & 18 & 0.21332 & 0.22572 \\
\hline $\mathrm{H}$ & 19 & 0.20323 & 0.22284 & $\mathrm{H}$ & 19 & 0.20308 & 0.20965 \\
\hline $\mathrm{H}$ & 20 & 0.21257 & 0.21010 & $\mathrm{H}$ & 20 & 0.39369 & 0.43416 \\
\hline $\mathrm{H}$ & 21 & 0.20261 & 0.20438 & $\mathrm{H}$ & 21 & 0.20116 & 0.20670 \\
\hline $\mathrm{H}$ & 22 & 0.19749 & 0.20488 & $\mathrm{H}$ & 22 & 0.20066 & 0.20688 \\
\hline $\mathrm{H}$ & 23 & 0.19832 & 0.20443 & $\mathrm{H}$ & 23 & 0.20226 & 0.21088 \\
\hline $\mathrm{H}$ & 24 & 0.19631 & 0.21959 & $\mathrm{H}$ & 24 & 0.20361 & 0.21404 \\
\hline $\mathrm{H}$ & 25 & 0.20839 & 0.24001 & $\mathrm{H}$ & 25 & 0.19970 & 0.20656 \\
\hline $\mathrm{H}$ & 26 & 0.22463 & 0.24316 & $\mathrm{H}$ & 26 & 0.20449 & 0.20907 \\
\hline $\mathrm{H}$ & 27 & 0.21197 & 0.20993 & $\mathrm{H}$ & 27 & 0.20236 & 0.21033 \\
\hline $\mathrm{C}$ & 28 & & 0.91933 & $\mathrm{H}$ & 28 & 0.19920 & 0.21376 \\
\hline $\mathrm{O}$ & 29 & & -0.75563 & $\mathrm{H}$ & 29 & 0.20648 & 0.20887 \\
\hline \multirow[t]{13}{*}{$\mathrm{O}$} & 30 & & -0.76164 & $\mathrm{H}$ & 30 & 0.20704 & 0.22184 \\
\hline & & & & $\mathrm{H}$ & 31 & 0.20033 & 0.21044 \\
\hline & & & & $\mathrm{H}$ & 32 & 0.19697 & 0.20719 \\
\hline & & & & $\mathrm{H}$ & 33 & 0.20958 & 0.22564 \\
\hline & & & & $\mathrm{H}$ & 34 & 0.19729 & 0.20443 \\
\hline & & & & $\mathrm{H}$ & 35 & 0.19936 & 0.22490 \\
\hline & & & & $\mathrm{H}$ & 36 & 0.19597 & 0.20825 \\
\hline & & & & $\mathrm{H}$ & 37 & 0.19502 & 0.20428 \\
\hline & & & & $\mathrm{H}$ & 38 & 0.20107 & 0.23017 \\
\hline & & & & $\mathrm{H}$ & 39 & 0.19659 & 0.19825 \\
\hline & & & & $\mathrm{C}$ & 40 & & 0.93327 \\
\hline & & & & $\mathrm{O}$ & 41 & & -0.72774 \\
\hline & & & & $\mathrm{O}$ & 42 & & -0.77949 \\
\hline
\end{tabular}


6. Cartesian coordinates and total energies for the calculated molecules

\begin{tabular}{|c|c|c|}
\hline Molecule & Base & Total Energy (a.u.) \\
\hline$\overline{\mathrm{DBU}}$ & $6-311+g^{*}$ & $-462,2001$ \\
\hline $\mathrm{DBU}-\mathrm{CO}_{2}$ & $6-311+g^{*}$ & $-650,8543$ \\
\hline PMDBD & $6-311+g^{*}$ & $-619,5065$ \\
\hline PMDBD- $\mathrm{CO}_{2}$ & $6-311+g^{*}$ & $-808,1520$ \\
\hline $\mathrm{CO}_{2}$ & $6-311+g^{*}$ & -188.5770 \\
\hline Ethanol & $6-311+g^{*}$ & -155.1924 \\
\hline DBU & cc-pVTZ & $-462,2558$ \\
\hline $\mathrm{DBU}-\mathrm{CO}_{2}$ & $c c-p V T Z$ & $-650,9133$ \\
\hline PMDBD & cc-pVTZ & $-619,5876$ \\
\hline $\mathrm{PMDBD}-\mathrm{CO}_{2}$ & cc-pVTZ & $-808,2449$ \\
\hline $\mathrm{CO}_{2}$ & cc-pVTZ & -188.5898 \\
\hline Ethanol & cc-pVTZ & -155.1448 \\
\hline
\end{tabular}

DFT with solvent (acetonitrile) - Ethanol- Cartesian coordinates

\begin{tabular}{|c|c|c|c|}
\hline & & & \\
\hline $\mathrm{C}$ & -0.290858 & 0.592022 & -1.056346 \\
\hline $\mathrm{C}$ & -0.168505 & 0.310828 & 0.434869 \\
\hline $\mathrm{H}$ & 0.691521 & 0.606183 & -1.539528 \\
\hline $\mathrm{H}$ & -0.902843 & -0.168985 & -1.548400 \\
\hline $\mathrm{H}$ & -0.758287 & 1.568003 & -1.223689 \\
\hline $\mathrm{H}$ & -1.155147 & 0.305631 & 0.904622 \\
\hline $\mathrm{H}$ & 0.425566 & 1.093073 & 0.924977 \\
\hline $\mathrm{O}$ & 0.390630 & -0.977950 & 0.720199 \\
\hline $\mathrm{H}$ & 1.330328 & -0.997397 & 0.449283 \\
\hline
\end{tabular}

DFT with solvent $-\mathrm{CO}_{2}-$ Cartesian coordinates

01

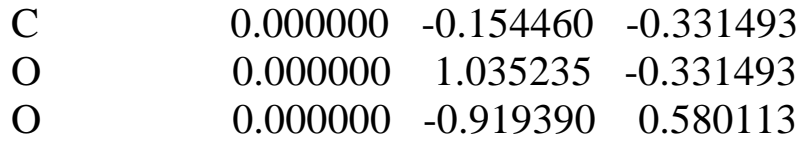


DFT with solvent (acetonitrile) - DBU - Cartesian coordinates

$\begin{array}{lrrr}0 & & & \\ \mathrm{C} & 0.000000 & 0.000000 & 0.000000 \\ \mathrm{C} & 0.000000 & 0.000000 & 1.519971 \\ \mathrm{C} & 1.444265 & 0.000000 & 2.005787 \\ \mathrm{~N} & 2.255411 & -1.046037 & 1.382679 \\ \mathrm{C} & 1.932845 & -1.492884 & 0.209734 \\ \mathrm{~N} & 0.829197 & -1.094283 & -0.526366 \\ \mathrm{C} & 0.675916 & -1.260167 & -1.985330 \\ \mathrm{C} & 0.975519 & -2.647199 & -2.559421 \\ \mathrm{C} & 2.459976 & -2.905053 & -2.855138 \\ \mathrm{C} & 3.388568 & -2.379483 & -1.751540 \\ \mathrm{C} & 2.829475 & -2.597025 & -0.334124 \\ \mathrm{H} & 0.363618 & 0.966514 & -0.381127 \\ \mathrm{H} & -1.014688 & -0.133983 & -0.386758 \\ \mathrm{H} & -0.517829 & -0.893079 & 1.888391 \\ \mathrm{H} & -0.546465 & 0.871930 & 1.893477 \\ \mathrm{H} & 1.488094 & -0.138910 & 3.091261 \\ \mathrm{H} & 1.906316 & 0.977870 & 1.807437 \\ \mathrm{H} & 1.263928 & -0.494758 & -2.515456 \\ \mathrm{H} & -0.372392 & -1.028427 & -2.188252 \\ \mathrm{H} & 0.402248 & -2.753126 & -3.486797 \\ \mathrm{H} & 0.577236 & -3.403558 & -1.875077 \\ \mathrm{H} & 2.732769 & -2.441443 & -3.810345 \\ \mathrm{H} & 2.611774 & -3.983123 & -2.984423 \\ \mathrm{H} & 3.588105 & -1.311806 & -1.900892 \\ \mathrm{H} & 4.361947 & -2.874365 & -1.829656 \\ \mathrm{H} & 3.647436 & -2.670332 & 0.382561 \\ \mathrm{H} & 2.293674 & -3.553403 & -0.287671\end{array}$

DFT with solvent (acetonitrile)- DBU- $\mathrm{CO}_{2}$ - Cartesian coordinates

$\begin{array}{lrrr}0 & & & \\ \mathrm{C} & 2.415025 & -0.117544 & -0.063849 \\ \mathrm{C} & 2.398430 & 0.343185 & 1.379486 \\ \mathrm{C} & 1.247968 & -0.330970 & 2.099267 \\ \mathrm{~N} & -0.004075 & -0.070254 & 1.365459 \\ \mathrm{C} & -0.046297 & 0.000810 & 0.034033 \\ \mathrm{~N} & 1.077979 & -0.023621 & -0.696745 \\ \mathrm{C} & 1.155530 & -0.151303 & -2.178991 \\ \mathrm{C} & 0.137442 & 0.645804 & -2.993274 \\ \mathrm{C} & -1.203231 & -0.077191 & -3.162456 \\ \mathrm{C} & -1.631465 & -0.782178 & -1.872794 \\ \mathrm{C} & -1.418866 & 0.077531 & -0.613966 \\ \mathrm{H} & 2.760294 & -1.155264 & -0.142497\end{array}$




$\begin{array}{lrrr}\mathrm{H} & 3.095199 & 0.499923 & -0.653588 \\ \mathrm{H} & 2.284136 & 1.430669 & 1.430864 \\ \mathrm{H} & 3.349122 & 0.086827 & 1.854155 \\ \mathrm{H} & 1.126982 & 0.054732 & 3.110819 \\ \mathrm{H} & 1.125024 & -1.215649 & -2.440420 \\ \mathrm{H} & 2.155636 & 0.199761 & -2.432719 \\ \mathrm{H} & 0.582281 & 0.838241 & -3.974739 \\ \mathrm{H} & 0.001560 & 1.628899 & -2.530345 \\ \mathrm{H} & -1.132023 & -0.813053 & -3.971019 \\ \mathrm{H} & -1.969153 & 0.645273 & -3.465377 \\ \mathrm{H} & -1.107352 & -1.737760 & -1.762413 \\ \mathrm{H} & -2.694263 & -1.035592 & -1.925763 \\ \mathrm{H} & -2.143033 & -0.225801 & 0.136546 \\ \mathrm{H} & -1.643712 & 1.129244 & -0.822131 \\ \mathrm{H} & 1.401530 & -1.412380 & 2.175042 \\ \mathrm{C} & -1.223006 & 0.134691 & 2.267487 \\ \mathrm{O} & -1.857549 & 1.175550 & 2.064309 \\ \mathrm{O} & -1.354793 & -0.765295 & 3.106057\end{array}$

PMDBD - Cartesian coordinates

$\begin{array}{lrrr}01 & & & \\ \mathrm{C} & 1.455805 & 0.942768 & 1.222930 \\ \mathrm{C} & 0.932556 & 0.319989 & 2.518269 \\ \mathrm{C} & -0.563924 & -0.031144 & 2.440244 \\ \mathrm{~N} & -0.817227 & -0.765384 & 1.173183 \\ \mathrm{C} & -0.294288 & -0.337944 & -0.045860 \\ \mathrm{C} & 1.186076 & 0.060771 & -0.017077 \\ \mathrm{C} & -1.435940 & 1.240875 & 2.501210 \\ \mathrm{C} & -0.946336 & -0.960918 & 3.598220 \\ \mathrm{C} & 2.045360 & -1.225168 & 0.060088 \\ \mathrm{C} & 1.519055 & 0.840389 & -1.302087 \\ \mathrm{C} & 0.950570 & 0.143297 & -2.535907 \\ \mathrm{C} & -0.580706 & -0.045607 & -2.433008 \\ \mathrm{~N} & -1.052556 & -0.394764 & -1.080216 \\ \mathrm{C} & -1.021751 & -1.172866 & -3.381305 \\ \mathrm{C} & -1.306809 & 1.253582 & -2.837826 \\ \mathrm{H} & 2.534397 & 1.122671 & 1.302096 \\ \mathrm{H} & 0.995489 & 1.925202 & 1.068723 \\ \mathrm{H} & 1.091436 & 1.006287 & 3.356665\end{array}$




$\begin{array}{lrrr}\mathrm{H} & 1.498490 & -0.586959 & 2.752540 \\ \mathrm{H} & -1.802494 & -1.020220 & 1.066133 \\ \mathrm{H} & -1.211643 & 1.928042 & 1.681922 \\ \mathrm{H} & -2.496911 & 0.981318 & 2.431601 \\ \mathrm{H} & -1.283984 & 1.775830 & 3.443749 \\ \mathrm{H} & -0.773423 & -0.470952 & 4.560740 \\ \mathrm{H} & -2.005583 & -1.234390 & 3.550596 \\ \mathrm{H} & -0.356846 & -1.880675 & 3.568010 \\ \mathrm{H} & 1.901521 & -1.861578 & -0.815232 \\ \mathrm{H} & 3.106043 & -0.958182 & 0.111098 \\ \mathrm{H} & 1.803578 & -1.825382 & 0.937630 \\ \mathrm{H} & 2.605918 & 0.953070 & -1.387450 \\ \mathrm{H} & 1.110323 & 1.854655 & -1.225590 \\ \mathrm{H} & 1.429788 & -0.833218 & -2.662655 \\ \mathrm{H} & 1.186622 & 0.714074 & -3.440664 \\ \mathrm{H} & -0.570284 & -2.125263 & -3.088265 \\ \mathrm{H} & -2.108528 & -1.293589 & -3.360294 \\ \mathrm{H} & -0.720475 & -0.957037 & -4.411532 \\ \mathrm{H} & -1.102537 & 1.504569 & -3.883422 \\ \mathrm{H} & -2.387140 & 1.137570 & -2.717752 \\ \mathrm{H} & -0.993287 & 2.097051 & -2.216768\end{array}$

Final structure - PMDBD- $\mathrm{CO}_{2}-$ Cartesian coordinates

$\begin{array}{lrrr}0 & & & \\ \mathrm{C} & -1.725550 & -1.796771 & 0.685516 \\ \mathrm{C} & -2.922828 & -1.000464 & 0.176492 \\ \mathrm{C} & -2.640662 & 0.510558 & 0.149844 \\ \mathrm{~N} & -1.213528 & 0.784217 & -0.174989 \\ \mathrm{C} & -0.157604 & -0.018014 & -0.117648 \\ \mathrm{C} & -0.443364 & -1.520232 & -0.127680 \\ \mathrm{C} & -2.908869 & 1.144340 & 1.528858 \\ \mathrm{C} & -3.496545 & 1.206811 & -0.916348 \\ \mathrm{C} & -0.656826 & -1.925590 & -1.612346 \\ \mathrm{C} & 0.725879 & -2.312278 & 0.464111 \\ \mathrm{C} & 2.038542 & -1.854088 & -0.144541 \\ \mathrm{C} & 2.335082 & -0.364831 & 0.111418 \\ \mathrm{~N} & 1.092342 & 0.491027 & -0.122514 \\ \mathrm{C} & 3.444195 & 0.048074 & -0.871040 \\ \mathrm{C} & 2.796488 & -0.187316 & 1.571421 \\ \mathrm{H} & -1.938862 & -2.869068 & 0.632586 \\ \mathrm{H} & -1.534464 & -1.572808 & 1.741124 \\ \mathrm{H} & -3.794846 & -1.180593 & 0.811435\end{array}$




$\begin{array}{lrrr}\mathrm{H} & -3.201544 & -1.343825 & -0.822829 \\ \mathrm{H} & -0.969177 & 1.762729 & -0.352189 \\ \mathrm{H} & -2.339144 & 0.643471 & 2.315316 \\ \mathrm{H} & -2.631186 & 2.201900 & 1.532754 \\ \mathrm{H} & -3.971203 & 1.072756 & 1.776008 \\ \mathrm{H} & -4.556806 & 1.037518 & -0.712508 \\ \mathrm{H} & -3.325918 & 2.287531 & -0.921189 \\ \mathrm{H} & -3.273213 & 0.819552 & -1.913275 \\ \mathrm{H} & 0.241777 & -1.779721 & -2.211795 \\ \mathrm{H} & -0.924003 & -2.985123 & -1.655982 \\ \mathrm{H} & -1.453990 & -1.355069 & -2.087505 \\ \mathrm{H} & 0.562750 & -3.377558 & 0.272892 \\ \mathrm{H} & 0.740880 & -2.193742 & 1.552380 \\ \mathrm{H} & 2.046500 & -2.052924 & -1.219633 \\ \mathrm{H} & 2.873414 & -2.425104 & 0.271002 \\ \mathrm{H} & 3.071182 & 0.032474 & -1.899081 \\ \mathrm{H} & 3.838214 & 1.036705 & -0.655382 \\ \mathrm{H} & 4.261084 & -0.675476 & -0.803119 \\ \mathrm{H} & 3.701300 & -0.778364 & 1.739589 \\ \mathrm{H} & 3.014141 & 0.854987 & 1.782571 \\ \mathrm{H} & 2.035390 & -0.533214 & 2.275190 \\ \mathrm{C} & 1.316590 & 2.027431 & -0.191556 \\ \mathrm{O} & 2.346244 & 2.430363 & 0.335694 \\ \mathrm{O} & 0.422364 & 2.657196 & -0.786300\end{array}$

7. General analytical details and compounds characterization.

The ${ }^{1} \mathrm{H}$ and ${ }^{13} \mathrm{C}$ NMR spectra were obtained with a Bruker AC 200 instrument, using TMS as internal standard and $\mathrm{CDCl}_{3}$ or $\mathrm{D}_{2} \mathrm{O}$ as solvents.

The chromatographic analyses were performed by HRGC using a HP 5890 gas chromatograph equipped with a flame ionization detector and a HP-5 column (25 m x 0.20 $\mathrm{mm} \times 0.33 \mathrm{~mm})$. The temperature of both the injector and the detector were fixed at $300{ }^{\circ} \mathrm{C}$. The GC-MS analyses were carried out using a HP 5890 gas chromatograph coupled with a HP 5970 Series Mass Selective Detector, using a HP-1 column (50 m x $0.20 \mathrm{~mm}$ x $0.33 \mathrm{~mm}$ ). The temperatures of both the injector and the interface were set at $300{ }^{\circ} \mathrm{C}$.

The elemental analyses were performed on an EA 1110 analyzer from CE Instruments. 
The TGA were performed using a TA Instruments TGA 2950 DSC 2910 calorimeter. The dynamic flow of $\mathrm{N}_{2}$ was $90 \mathrm{~mL} \cdot \mathrm{min}^{-1}$ and the heating rate was $10^{\circ} \mathrm{C} \cdot \mathrm{min}^{-1}$ in the temperature range of 5 to $200^{\circ} \mathrm{C}$.

2-N-carboxyl-3,3,6,9,9-pentamethyldiazabicyclodecenium $\quad\left(\right.$ PMDBD-CO$\left._{2}\right)$. White powder: mp 77-80 ${ }^{\circ} \mathrm{C} .{ }^{1} \mathrm{H}$ NMR $\left(200.13 \mathrm{MHz}, \mathrm{CDCl}_{3}\right): \delta 1.22-1.30(\mathrm{~m}, 7 \mathrm{H}$, two methylen + methyl), 1.31-1.38 (m, 12H, four methyls), 1.57-1.97 (m, 4H, two methylene). ${ }^{13} \mathrm{C}$ NMR (50.32 MHz, $\left.\mathrm{CDCl}_{3}\right): \delta 24.6,29.7,30.9,31.4,31.9,34.7,53.3,162.2,166.0 . \mathrm{MS}(70 \mathrm{eV}): \mathrm{m} / \mathrm{z}$ 193(100\%), 194(20\%), 208( $\left.\mathrm{M}^{+}, 18 \%\right), 209(4 \%) . \mathrm{IR}(\mathrm{KBr}): \mathrm{cm}^{-1} 3566,3377,2977,2936$, 2666, 2375, 1667, 1647, 1576, 1468, 1402, 1382, 1335. Anal. Calcd. for $\mathrm{C}_{14} \mathrm{H}_{24} \mathrm{~N}_{2} \mathrm{O}_{2} \cdot \mathrm{H}_{2} \mathrm{O}: \mathrm{C}$, 62.22; H, 9.62; N, 10.37. Found: C, 61.82; H, 9.41; N, 10.47.

DBN-CO 2 complex. White crystalline powder. Hygroscopic and unstable compound, at 25 ${ }^{\circ} \mathrm{C}$. MS $(70 \mathrm{eV}): \mathrm{m} / \mathrm{z} 68,96,109,123(100 \%), 124\left(\mathrm{M}^{+}, 75 \%\right), 125(7 \%)$. Anal. Calcd. for $\mathrm{C}_{8} \mathrm{H}_{12} \mathrm{~N}_{2} \mathrm{O}_{2}$ : C, 57.14; H, 7.14; N, 16.66. Found: C, 57.32; H, 7.40; N, 16.43.

$N$-cyclohexyl ethyl carbamate. White solid: mp $122-124{ }^{\circ} \mathrm{C} .{ }^{1} \mathrm{H}$ NMR $(200.13 \mathrm{MHz}$, $\left.\mathrm{CDCl}_{3}\right): \delta$ 1,09- 1,27 (m, 9H, methylene + methyl); 1,60 (m, 2H, methylene); 1,91 (m, 2H, methylene ); 3,41 (m, 1H, CH ); 4,07 (q, 2H, methylene ); 4,53 (br s, 1H, NH). ${ }^{13} \mathrm{C}$ NMR (50.32 MHz, $\left.\mathrm{CDCl}_{3}\right):$ 14.5, 24.7, 25.4, 33.4, 49.6, 66.4, 155.8. MS (70 eV) m/z: 56(100 \%), 128, 142, 171(M $\left.\mathrm{M}^{+}\right)$. IR (KBr): $\mathrm{cm}^{-1} 1684,2855,2936,2983$, 3326. Anal. Calcd. for $\mathrm{C}_{9} \mathrm{H}_{17} \mathrm{NO}_{2}$ : C, 63.15; H, 9.94; N, 8.18. Found: C, 62.86; H, 10.10; N, 8.18. 
